\title{
Precise orbit determination of the Fengyun-3C satellite using onboard GPS and BDS observations
}

\author{
Min $\mathrm{Li}^{1,2}$ - Wenwen $\mathrm{Li}^{1}$ - Chuang $\mathrm{Shi}^{1,2}$ - Kecai Jiang ${ }^{1} \cdot \mathrm{Xiang} \mathrm{Guo}^{1}$. \\ Xiaolei Dai $^{1,2}$ - Xiangguang Meng ${ }^{3,4}$. Zhongdong Yang ${ }^{5}$. Guanglin Yang ${ }^{5}$. \\ Mi Liao ${ }^{5}$
}

Received: 2 September 2016 / Accepted: 10 April 2017 / Published online: 24 April 2017

(C) The Author(s) 2017. This article is an open access publication

\begin{abstract}
The GNSS Occultation Sounder instrument onboard the Chinese meteorological satellite Fengyun-3C (FY-3C) tracks both GPS and BDS signals for orbit determination. One month's worth of the onboard dual-frequency GPS and BDS data during March 2015 from the FY-3C satellite is analyzed in this study. The onboard BDS and GPS measurement quality is evaluated in terms of data quantity as well as code multipath error. Severe multipath errors for BDS code ranges are observed especially for high elevations for BDS medium earth orbit satellites (MEOs). The code multipath errors are estimated as piecewise linear model in $2^{\circ} \times 2^{\circ}$ grid and applied in precise orbit determination (POD) calculations. POD of FY-3C is firstly performed with GPS data, which shows orbit consistency of approximate $2.7 \mathrm{~cm}$ in 3D RMS (root mean square) by overlap comparisons; the estimated orbits are then used as reference orbits for evaluating the orbit precision of GPS and BDS combined POD as well as BDS-based POD. It is indicated that inclusion of BDS geosynchronous orbit satellites (GEOs) could degrade POD precision seriously. The precisions of orbit estimates by combined POD and BDS-based POD are 3.4 and $30.1 \mathrm{~cm}$ in
\end{abstract}

Wenwen Li

cheeselee@whu.edu.cn

1 GNSS Research Center, Wuhan University, 129 Luoyu Road, Wuhan 430079, China

2 Collaborative Innovation Center of Geospatial Technology, 129 Luoyu Road, Wuhan 430079, China

3 National Space Science Center, Chinese Academy of Sciences, 1 Nanertiao Road, Beijing 100190, China

4 Beijing Key Laboratory of Space Environment Exploration, 1 Nanertiao Road, Beijing 100190, China

5 National Satellite Meteorological Center, China Meteorological Administration, 46 Zhongguancun South Street, Beijing 100081, China
3D RMS when GEOs are involved, respectively. However, if BDS GEOs are excluded, the combined POD can reach similar precision with respect to GPS POD, showing orbit differences about $0.8 \mathrm{~cm}$, while the orbit precision of BDSbased POD can be improved to $8.4 \mathrm{~cm}$. These results indicate that the POD performance with onboard BDS data alone can reach precision better than $10 \mathrm{~cm}$ with only five BDS inclined geosynchronous satellite orbit satellites and three MEOs. As the GNOS receiver can only track six BDS satellites for orbit positioning at its maximum channel, it can be expected that the performance of POD with onboard BDS data can be further improved if more observations are generated without such restrictions.

Keywords BDS - GPS · Multipath error · Data quality · Precise orbit determination

\section{Introduction}

For many low earth orbit (LEO) scientific missions, such as the geodetic and oceanographic missions designed for measuring global sea level, determining the earth's gravity field, or sensing the atmosphere using occultation signals, the orbit precision is often required at the centimeter level, which makes precise orbit determination (POD) particularly challenging. Over the past two decades, many LEO satellites including TOPEX/POSEIDON, JASON, CHAMP, GRACE and GOCE have utilized onboard GPS technique for POD purpose. By providing numerous high-precision, high-sampling rate data with full-arc tracking availability, the onboard GPS technique can produce orbits at centimeterlevel precision. The TOPEX/POSEIDON satellite is one of the early missions with space-borne GPS receivers, and its orbit precision using GPS data is about $3-4 \mathrm{~cm}$ in its radial 
component, which is far better than the required precision (Tapley et al. 1994). The follow-on missions Jason-1 and Jason-2 carry BlackJack GPS receivers onboard as well. By validation with satellite laser ranging (SLR) data and the crossover points, POD with GPS data alone achieves 1-cm-level precision in the radial component for Jason-1 (Haines et al. 2004), and even sub-centimeter level for Jason2 (Bertiger et al. 2010; Lemoine et al. 2010). The POD precision using onboard GPS data for GRACE and GOCE also reaches about $2 \mathrm{~cm}$ in $3 \mathrm{D}$ root mean square (RMS) (Kang et al. 2006; Bock et al. 2014), fulfilling the requirements for gravity recovery studies. These results not only show the significant success of applying onboard GPS technique in LEO POD, but also indicate the great potential of onboard GNSS techniques with other systems, such as the BeiDou Navigation Satellite system (BDS).

Similar to the other GNSS systems such as GPS, GLONASS and GALILEO, BDS aims at providing global positioning, navigation and timing services. To date, the BDS constellation comprises a total of 19 operational satellites in orbit, including 14 satellites from the regional BeiDou system (BDS-2 or COMPASS) and 5 satellites from the global BeiDou system (BDS-3). The BDS-2 system initialized its regional service during December 2012 (http://www.beidou. gov.cn/), and since then, many studies relating to BDS have been carried out, such as BDS satellite POD (Dai et al. 2015; Deng et al. 2014; Guo et al. 2016b; Lou et al. 2014; Prange et al. 2015; Shi et al. 2012; Steigenberger et al. 2013; Zhao et al. 2013), BDS for precise positioning (Cai et al. 2015; Li et al. 2014, 2015b; Prange et al. 2015), as well as BDS for troposphere sounding ( $\mathrm{Li}$ et al. 2015a; Lu et al. 2015; Xu et al. 2013) and ionosphere modeling (Zhang et al. 2015). These investigations indicate that the BDS precision products from different analysis centers agree on sub-meter level for BDS IGSOs (inclined geosynchronous satellite orbit satellites) and MEOs, but differ with each other by several meters for GEOs (Guo et al. 2016a, b). Based on these products, BDS PPP (Precise Point Positioning) in current BDS service area can achieve 1.0-cm-level precision in horizontal component and better than $3.0-\mathrm{cm}-$ level precision in vertical component (Li et al. 2014, 2015a). The troposphere zenith path delay (ZTD) estimates from the ground-based BDS observables are broadly consistent with the GPS ZTD estimates, and their differences are generally about 11-16 mm (Lu et al. 2015). These results indicate that in its service area, BDS can now provide comparable precisions in position and ZTD estimates with respect to GPS. However, all these studies are carried out using ground-based data, while the quality of onboard BDS data and its contribution to LEO POD have not been investigated yet. As the BDS constellation is still under construction and the expected service area is mainly in the Asia and Pacific Ocean regions, the degree of precision that can be obtained for a LEO satellite with onboard BDS data remains unknown.

The onboard BDS data quality as well as the POD performance using onboard BDS data is of great interest to the geodesy community. Several simulation studies have already been performed to investigate the BDS-based LEO POD precision. Zhao et al. (2015) analyzed the BDS data coverage and positioning accuracy with simulation scenarios based on the China's Tiangong-1 spacelab and International Space Station, and indicated that the positioning using onboard BDS data only was valid for $32 \mathrm{~min}$ per orbit revolution (90 min) for Tiangong-1, and the positioning accuracy was about 10 $\mathrm{m}$ with an average position dilution of precision (PDOP) of 1.9 (Zhao et al. 2015). Liu et al. (2014) simulated the onboard BDS data with 14 BDS-2 satellites and considered the BDS orbit errors and the observation noise in the observation simulation setup. The resultant POD precision was $30 \mathrm{~cm}$ in 3D RMS (Liu et al. 2014). Chen et al. (2016) first reported the preliminary in-orbit results of the onboard BDS data from the Chinese Ling-Qiao satellite (Chen et al. 2016), but mainly focused on the BDS signal quality and the use of BDS code range measurements for kinematic positioning, rather than phase measurements for POD. The results indicated that positioning and velocity RMS with BDS were 13 $\mathrm{m}$ and $20 \mathrm{~cm} / \mathrm{s}$, respectively, while with GPS they were $1 \mathrm{~m}$ and $1.1 \mathrm{~cm} / \mathrm{s}$.

The Chinese FY-3C satellite, designed for collecting atmospheric data for intermediate- and long-term weather forecasting and global climate research (Bi et al. 2012), was launched in September 2013 with a sun-synchronous, nearcircular, near-earth orbit at an altitude of $836 \mathrm{~km}$. For both POD and occultation observing purposes, FY-3C carries a GNSS Occultation Sounder (GNOS) instrument onboard. The GNOS instrument can track both GPS and BDS-2 signals simultaneously and record dual-frequency code and carrier phase observations from both systems (Bai et al. 2014). These valuable GPS and BDS observations can provide opportunities for investigating the onboard data quality as well as the POD performances using these data.

Given this, the onboard dual-frequency GPS and BDS data from the FY-3C satellite are collected for the entire month of March 2015 and analyzed in this study. The FY-3C platform and the GNOS instrument are introduced and described in Sect. 2. In Sect. 3, the onboard GPS and BDS data quality is then evaluated in terms of data quantity, distribution patterns as well as multipath error. In Sect. 4, FY-3C precision orbits are determined with GPS-only, GPS and BDS combined as well as BDS-only observations by dynamic POD, respectively. The orbit consistency of GPS-based POD are analyzed by methods of residual analysis and orbit overlap comparisons, while the orbit precision of GPS and BDS combined POD as well as BDS-based POD is evaluated by orbit comparison with the GPS-derived orbits. 


\section{FY-3C platform and GNOS instrument description}

\subsection{FY-3C platform}

The Chinese Fengyun-3 (FY-3) satellites are the secondgeneration polar orbit meteorological satellites of China, while FY-3C is the first operational satellite for the FY-3 series, possessing 12 different payloads and sensors that can enhance its sounding and imaging capabilities significantly (Liao et al. 2016).

The FY-3C spacecraft structure in general is a hexahedron of $4.4 \mathrm{~m} \times 2.0 \mathrm{~m} \times 2.0 \mathrm{~m}$ in the stowed configuration and 4.4 $\mathrm{m} \times 10 \mathrm{~m} \times 3.8 \mathrm{~m}$ in the deployed state (Yang et al. 2012). The total spacecraft mass is $2450 \mathrm{~kg}$ before launching and $2405.7 \mathrm{~kg}$ thereafter, as provided by the Center for Space Science and Applied Research (CSSAR). FY-3C has only one solar panel mounted on the one side of the main body of the satellite for capturing solar energy.

The satellite body-fixed (SBF) frame of the FY-3C spacecraft is defined as follows: The origin of the SBF frame is the center of mass (COM) of the satellite; the $+\mathrm{X}$ axis points along the satellite velocity direction and the $+Z$ axis points toward the earth's center, while the $Y$ axis completes a righthand coordinate system with $\mathrm{Z}=\mathrm{X} \times \mathrm{Y}$. As provided by the CSSAR, the phase center offset (PCO) of the positioning antenna (PA) is measured as $(-1.280,0.282,-0.969) \mathrm{m}$ for $\mathrm{L} 1$ frequency signal and $(-1.278,0.282,-0.969) \mathrm{m}$ for L2 frequency signal, with respect to the COM in SBF frame.

The FY-3C follows a strict attitude control regime with a three-axis stabilization (Yang et al. 2012). In normal cases, the pitch, roll and yaw angles measured by the attitude determination and control subsystem (ADCS) are usually within $0.06^{\circ}$, indicating that the largest error attributing to $\mathrm{PCO}$ miscalculation is less than $2 \mathrm{~mm}$ in the line of sight (LOS) direction when using the nominal attitude model instead of the measured angles.

\subsection{GNOS instrument}

The initial GNOS instrument onboard the FY-3C satellite was developed by the CSSAR of the CAS (Chinese Academy of Sciences), Beijing, China. It contains three antennas, namely the PA (positioning antenna) and two sets of OAs (occultation antenna) for both rising and setting occultation signals in physical structure (Bai et al. 2014). For POD data analysis, the performance of the PA is of primary interest. The PA constitutes a wide beam with hemispherical coverage and low-gain patch antenna pointing into the zenith direction. For GPS signal, the GNOS instrument allocates eight channels for the PA and six for the OAs. However, the PA can also utilize the idle channels pre-assigned for the OA, such that the GPS satellite number at some epochs can be greater than eight. For the BDS signal, there are four fixed channels allocated for the PA and four channels for the OAs. Similarly, the PA for the BDS signal can also use up to two idle channels from those pre-assigned for the BDS occultation signal, which means the BDS satellite number at one single epoch can be more than four, but no larger than six.

The PA of the GNOS tracks the BDS B1/B2 frequency signals and the GPS L1/L2 signals, through which it can provide multifrequency code, carrier phase and signal-noise ratio (SNR) observations with BDS/GPS compatibility (Bai et al. 2014). The supported signal types are L1 C/A, L2C and L2P for GPS and B1I and B2I for BDS. It should be noted that GNOS utilizes the GPS L2 semi-codeless method operating for $\mathrm{P}$ code to maintain stable tracking. Wang et al. (2014) and Bai et al. (2014) analyzed the code and carrier phase measurement precision of the GNOS instrument in a laboratory with a Spirent GSS8000 BDS and GPS signal simulator, which indicated that the pseudorange precision of GNOS is less than $17 \mathrm{~cm}$ for BDS B1 code observations, 30 $\mathrm{cm}$ for BDS B2 and $16 \mathrm{~cm}$ for GPS, while the carrier phase precision is less than $1.2 \mathrm{~mm}$ for both BDS and GPS.

\section{Data collection and quality evaluation}

In this section, the data source for this study is first introduced. Evaluation of the real onboard BDS and GPS data quality is then performed mainly in terms of data quantity, distribution characteristics and multipath error. The obtained results are then analyzed and discussed.

\subsection{Data collection}

The FY-3C onboard GPS and BDS data from March 1 to 31, 2015, are collected from National Satellite Meteorological Center and then employed in this study. Both the GPS and BDS data are recorded in 1-s sampling rate. In accordance with the RINEX-3 observation code definition (IGS 2015), the provided data include GPS L1 frequency code range and carrier phase measurements C1C and L1C, GPS L2 observations $\mathrm{C} 2 \mathrm{P}$ and $\mathrm{L} 2 \mathrm{P}, \mathrm{BDS} \mathrm{B} 1$ frequency data $\mathrm{C} 2 \mathrm{I}$ and L2I, as well as BDS B2 frequency data C7I and L7I.

In order to perform POD, precise GPS and BDS orbit and clock products are needed to introduce in high-precision time and coordinate frames. The final GPS orbit and 30-s clock products distributed by the International GNSS Service (IGS) are used for the GPS-based FY-3C POD. These orbit and clock products are generated using a combination of products from different analysis centers (ACs), and are generally recognized as the best quality. Specifically, the GPS orbit accuracy of the IGS final precise orbit products can reach $2.5 \mathrm{~cm}$ in 1D RMS and clock products reach $75 \mathrm{ps}$ in RMS (Dow et al. 2009). The 30-s clock products are used to 
avoid the precision loss due to interpolation since a typical data processing interval of $30 \mathrm{~s}$ is used for POD in this study.

For the GPS and BDS combined POD as well as BDSbased POD, the precise BDS orbit and clock products are also required. Currently, different MGEX (Multi-GNSS Experiment) ACs including CODE (Center for Orbit Determination in Europe), GFZ (GeoForschungsZentrum Potsdam) and Wuhan University provide the BDS precise orbit and clock products routinely (Prange et al. 2015; Uhlemann et al. 2015; Guo et al. 2016b). Guo et al. (2016a,b) evaluated the qualities of these products. The independent orbit comparisons indicated that the orbit agreement between the different ACs can reach about $0.2-0.3 \mathrm{~m}$ for BDS IGSO and $0.1-0.2 \mathrm{~m}$ for BDS MEO satellites in 3D RMS, while for BDS GEO the differences can reach several meters. The BDS clock comparisons show an agreement of $0.5-0.8 \mathrm{~ns}, 0.2-0.3 \mathrm{~ns}$ and $0.15-0.2$ ns for GEO, IGSO and MEO satellites, respectively. However, the MGEX clock products from both Wuhan University and GFZ during March 2015 are provided in 300-s sampling interval, which could result in significant precision loss when interpolated to $30 \mathrm{~s}$. So the recomputed 30-s interval BDS clock and orbit products by Wuhan University have been adopted. These products are actually generated using the same strategy as the products submitted to MGEX, and thus, their precisions are comparable. In addition, since a two-step method is used to generate the BDS products, during which the station coordinates and ZTDs are firstly estimated using IGS products and then fixed for BDS satellite POD (Dr. Jing Guo, personal communication), the frame differences between the IGS final products and the recomputed BDS products are very small and can be neglected in subsequent data processing and analysis.

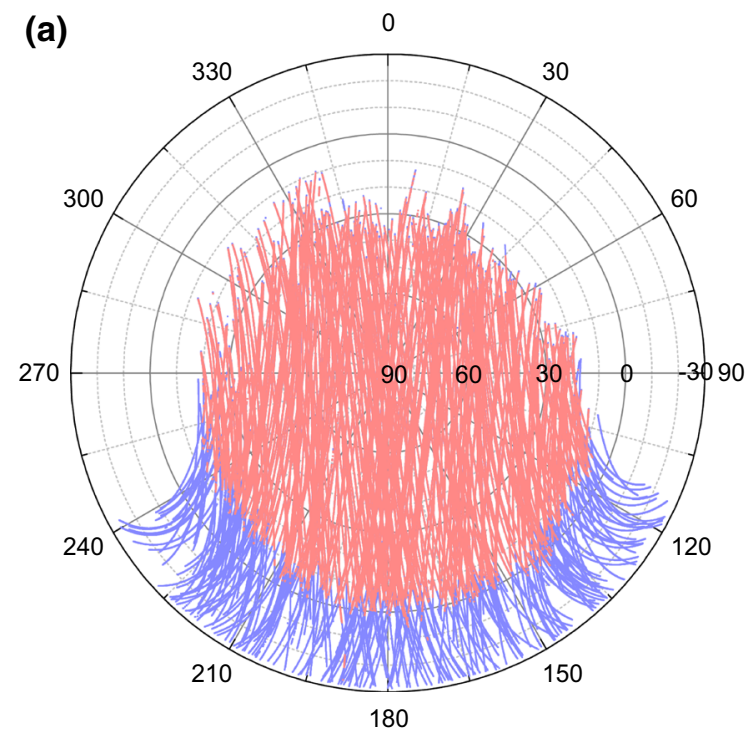

\subsection{Onboard data distribution characters}

As the POD calculations utilize the un-differenced, ionosphere-free carrier phase and code linear combination as basic observation equations, both code and carrier phase observations from two frequencies of GPS or BDS are needed at each single epoch. In the following text, we refer to a usable epoch or satellite only if all four types of observations are available for that epoch or satellite. The measurements with an elevation angle less than 0 are discarded, as they are generally discarded in POD data processing due to their lower precision as a result of amplified multipath errors.

For BDS, the numbers of the four types of observations are almost the same, but generally the number of L2I/C2I is slightly higher than L7I/C7I. For GPS, there are obviously more $\mathrm{L} 1 \mathrm{C} / \mathrm{C} 1 \mathrm{C}$ observations than L2P/C2P. This could be attributed to the fact that GNOS uses a semi-codeless method for tracking the $\mathrm{C} 2 \mathrm{P}$ signal as the $\mathrm{P}$ code structure is encrypted. The semi-codeless technique involves signal multiplications that result in a lower SNR compared to a direct tracking, as well as a higher risk of signal loss of lock (Montenbruck et al. 2006).

To demonstrate the data distribution pattern seen from the antenna and the GPS L2 frequency data loss against L1 frequency data, the sky view of L1 and L2 frequency data points in the antenna reference frame (ARF) on DOY 064/2015 is indicated in Fig. 1a. As the PA is installed along the $-\mathrm{Z}$ direction in the SBF frame, the ARF here is defined as follows: The azimuth angle is counted from the $\mathrm{X}$ axis of the SBF frame, while the zenith is measured from the $-\mathrm{Z}$ direction. The GPS L2 measurements are mainly confined to a minimum elevation angle of $0^{\circ}$, while $\mathrm{L} 1$ are actually tracked

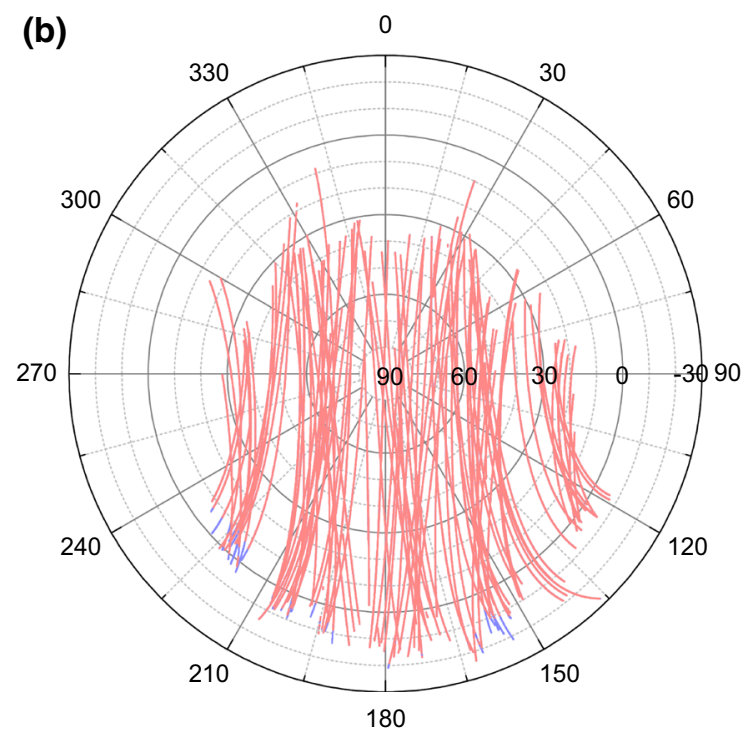

Fig. 1 Sky view of GPS L1/L2 as well as BDS B1/B2 data points in ARF. Blue dots represent the L1 or B1 data while red represent L2 or B2. a GPS data; b BDS data 


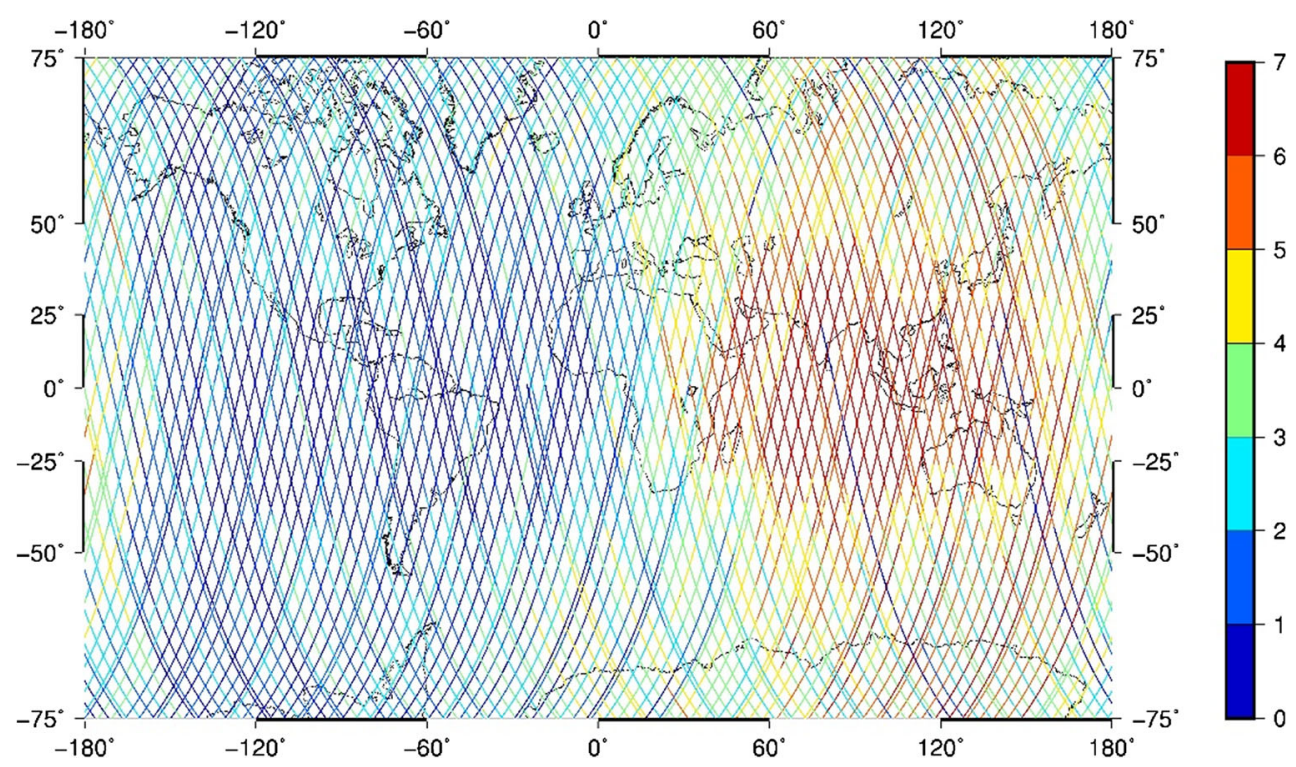

Fig. 2 Number of observed useful BDS satellites per epoch along FY-3C satellite ground tracks from DOY 062/2015 to 067/2015. Different colors indicate the different satellite numbers

far below the horizontal plane reaching down to $-30^{\circ}$ in the setting passes. There are almost no observations in the fore hemisphere within $0-30^{\circ}$ elevation, but data coverage in the aft hemisphere is near complete, which is mainly attributed to the signal blockage by the microwave humidity sounder in the fore hemisphere. The loss of the L2 observations with respect to $\mathrm{L} 1$ is mainly in the aft hemisphere when elevation angle is less than $10-20^{\circ}$ and is near-symmetrical distributed in horizontal plane, as the SNR of L2 frequency decreases sharply with low elevations. This is similar to earlier LEO missions such as CHAMP (Montenbruck and Kroes 2003) and HY-2A (Guo et al. 2015), for which the onboard GPS data also indicated loss of L2 data that is dependent on elevations and near-symmetrical distribution along the flight direction. The sky plot for the BDS data shown in Fig. 1b indicates that the numbers of $\mathrm{B} 1$ and $\mathrm{B} 2$ frequency data are almost the same, and only a small amount of B2 data is lost in the aft hemisphere. The minimum elevation for the BDS signal is about $-20^{\circ}$, which is a little larger than that of the GPS. Like the GPS data, the distribution of BDS data in the fore hemisphere is not as complete as that in the aft hemisphere.

Due to the BDS constellation configuration in March 2015, the data distribution along the satellite ground tracks is very unbalanced globally. The observed BDS satellite numbers along the FY-3C satellite orbit from DOY 062/2015 to $067 / 2015$ are shown in Fig. 2. A six-day arc is used for this analysis since the regression period for the FY-3C is typically 5.5 days. It is evident that there are very few observed satellites in the Western Hemisphere, and mostly only zero to two satellites can be used. However, the number of observed satellites in the Eastern Hemisphere is obviously larger than that in the Western Hemisphere, comprising three to six in most cases. Almost all the epochs can have six usable satellites in the Asia-Pacific Ocean region, reaching the maximum BDS channels allocated for PA, which is exactly the standard service area for BDS-2. In fact, the number of BDS satellites that can be observed in the BDS service area can easily reach ten or more, according to the ground-based BDS data as well as the space-borne BDS receiver on the Ling-Qiao satellite (Chen et al. 2016). There are a few tracks showing no usable observations in the Eastern Hemisphere, which could be attributed to the receiver reset or receiver tracking problems. As the GNOS receiver clock error could reach about $3 \mathrm{~ms}$ on a daily basis by free steering, the GNOS is routinely reset approximately every $24.25 \mathrm{~h}$, resulting in temporal data outage. Figure 2 shows that $76.5 \%$ of the BDS observations are distributed in the Eastern Hemisphere (specifically the Asia and Indian Ocean region), leaving only $23.5 \%$ in Western Hemisphere.

Figure 3 shows the average percentages of number of useful GPS and BDS satellites per epoch. Severe data loss, with over one quarter of a day of useless data, is found for BDS on DOY 061, 076-078 and 080, as well as for GPS on DOY 074. These days are thus excluded from the statistics. For BDS, over $15 \%$ of the epochs possess useful satellites ranging from zero to three individually, while about $10 \%$ between four and six. The statistics for the GPS are quite different from the BDS, as for over $95 \%$ of epochs the number of useful satellites is greater than four. It also indicates that there are only about $2.6 \%$ epochs with no useful satellite. The maximum number of useful GPS satellites for one epoch can reach up to 12 . 


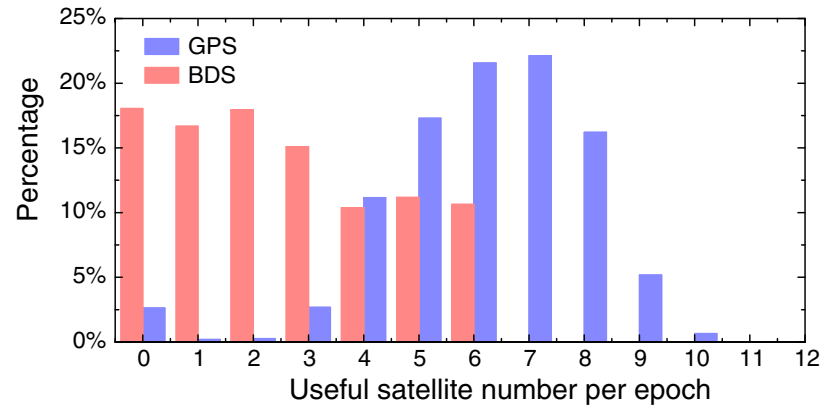

Fig. 3 Average percentages of the number of useful GPS and BDS satellites per epoch using one-month data. Red bars represent BDS observations and blue bars represent GPS. The days with serious data loss are not considered

\subsection{Multipath error}

Studies of the BDS multipath error have become of particular interest in recent years due to the presence of satellite-related code multipath biases in comparison with other GNSS systems, which can be well modeled as piecewise linear or polynomials associated with elevation angles for MEOs and IGSOs based on BDS data from ground stations (Wanninger and Beer 2015; Yang et al. 2016). However, with onboard tracking data, the observation coverage in the sky plot can be much more complete, which can help to further reveal the BDS multipath errors relating to elevations as well as azimuths. In this section, the multipath errors of onboard BDS/GPS data are analyzed.

The BDS and GPS code multipath errors are calculated using the multipath combinations with dual-frequency code and phase observations (e.g., Rocken and Meertens 1992; Wanninger and Beer 2015). In the below text, MP1 refers to the multipath errors of BDS B1 or GPS L1 pseudorange observations $(\mathrm{C} 2 \mathrm{I} / \mathrm{C} 1 \mathrm{C})$ while MP2 refers to the multipath errors of BDS C7I or GPS C2P observations.

In most cases, the BDS MP1 and MP2 for each satellite are within $\pm 2 \mathrm{~m}$, while the MP2 series reveals slightly smaller variations compared to MP1. Both BDS MP1 and MP2 indicate obvious deterioration with low elevation angles, becoming as large as $3-4 \mathrm{~m}$. The multipath variation patterns of FY-3C onboard BDS data differ from those observed from ground BDS data, which usually show elevation-dependent biases of MP1 and MP2 for each BDS IGSOs and MEOs in full satellite passes (Wanninger and Beer 2015). In Fig. 4, the upper panels illustrate the BDS C05 (GEO satellite), C07 (IGSO satellite) and C11 (MEO satellite) MP1 and elevation variations with respect to epoch on DOY 064/2015. For $\mathrm{C} 05$, the MP1 values are generally within $\pm 1 \mathrm{~m}$ and only small biases are observed. The C07 MP1 pattern is very similar to the results from ground BDS data, indicating obvious elevation-dependent biases in both rising and setting passes. However, there are noticeable high-frequency variations in the C07 MP1 series during the setting pass, which are also observed in the GPS multipath error series and coincide very well with the SNR variations. The scatters of C11 MP1 are most tightly clustered among the three satellites. For the rising pass of $\mathrm{C} 11$, the MP1 decrease nearly linearly, indicating significant elevation-dependent biases, while for the setting pass the MP1 variations are very consistent. In Fig. 4d-f, the MP1 values of C05, C07 and C11 are plotted against elevations to show their relationship. It can be seen that for all three satellites, significant multipath biases are only observed for elevations larger than $40^{\circ}$, while the multipath errors are distributed rather evenly for elevations between $0^{\circ}-40^{\circ}$.
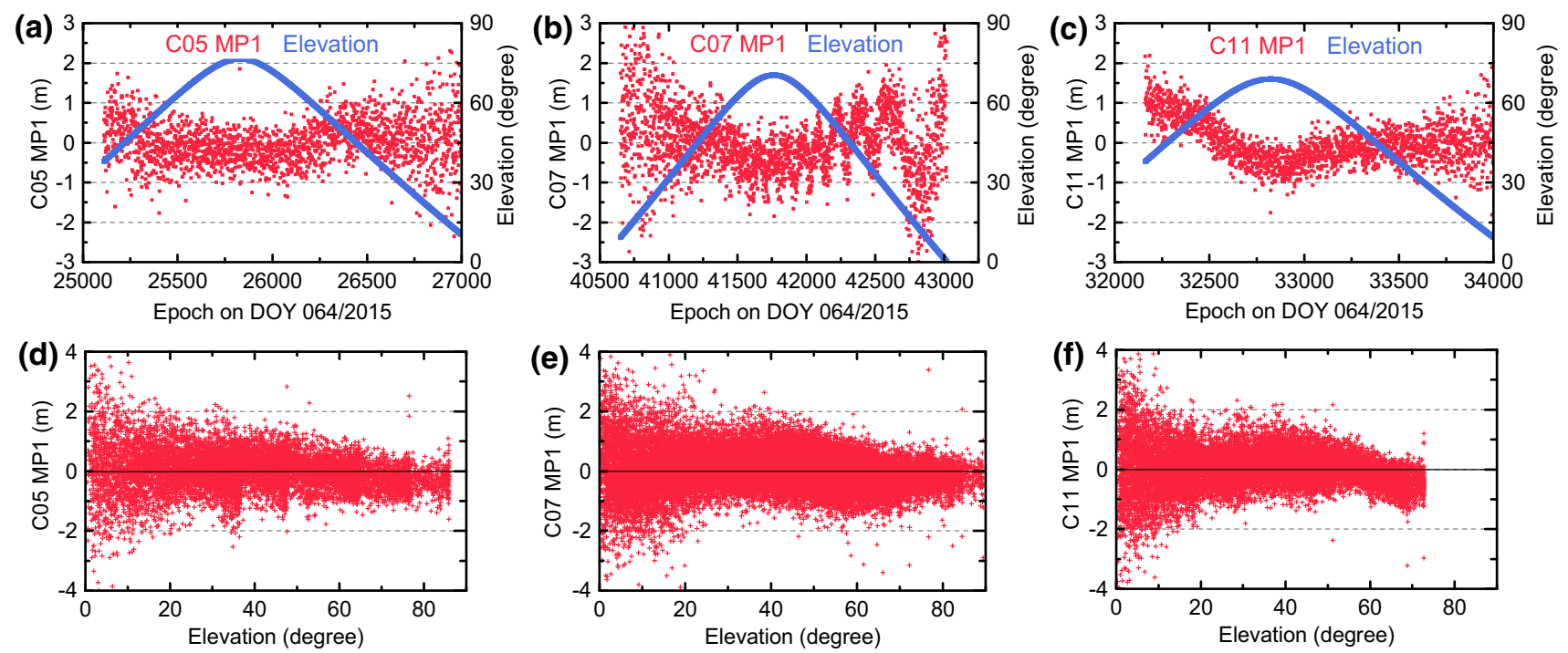

Fig. 4 The upper panels show the BDS C05 (GEO), C07 (IGSO) and C11 (MEO) MP1 time series and elevation variations with respect to epoch on DOY 064/2015, while the bottom panels illustrate the C05, C07 and C11 MP1 values associated with elevations on DOY 064/2015 
To further demonstrate the dependence of code multipath errors on both elevation and azimuth, the BDS and GPS MP1 and MP2 are modeled and estimated as piecewise linear model with a grid resolution of $2^{\circ} \times 2^{\circ}$. For BDS, we estimate code multipath maps for different constellations separately. As seen in Fig. 5, all BDS GEO, IGSO and MEO MP1 maps show significant systematic variations in the whole plane, which obviously are dependent on both elevations and azimuths and can reach around $1.0 \mathrm{~m}$ in the fore hemisphere. When elevations are larger than $60^{\circ}$, the MP1 errors are biased by about -0.2 to $-0.4 \mathrm{~m}$ for BDS GEOs and IGSOs, while even to $-1.0 \mathrm{~m}$ for MEOs. The BDS MP2 grid values are generally a little smaller than MP1 but show similar patterns. Significant MP2 biases are also observed for MEOs around $-0.5 \mathrm{~m}$ for elevations larger than $60^{\circ}$. Both BDS IGSOs and MEOs multipath errors show fluctuations in the aft hemisphere, which are nearly symmetrically distributed along the flight direction. Comparatively, the GPS MP1 and MP2 grid maps show no biases in higher elevations, but the multipath in the fore hemisphere are also larger than the aft hemisphere. Systematic fluctuations in the aft hemisphere are observed as well. Above results imply that the FY-3C BDS code observations suffer from not only BDS satellite-induced but also strong near-field multipath errors. These estimated multipath maps are later applied in the POD calculations to correct GPS and BDS code range errors.

The multipath RMS statistics for both BDS and GPS code ranges for each single day are calculated as indicators for the onboard BDS and GPS code observation precision. The daily BDS MP1 RMS is generally within $0.7-0.8 \mathrm{~m}$, and the MP2 is within $0.6-0.7 \mathrm{~m}$. For GPS, the MP1 RMS is within $0.36-0.38 \mathrm{~m}$, while MP2 RMS is within $0.6-0.8 \mathrm{~m}$, nearly double the MP1 RMS value. Furthermore, there is a noticeably larger MP1 and MP2 RMS on DOY 075/2015, indicating code observation precision degradation. As the ionosphere-free code combination (PC) observables are used in the subsequent POD calculations, the PC multipath (MPC) RMS is also computed for both BDS and GPS. The average BDS MP1, MP2 and MPC RMS values are 0.73, 0.67 and $2.03 \mathrm{~m}$, respectively, while for GPS they are $0.36,0.61$ and $1.29 \mathrm{~m}$, respectively.

\section{POD results and analysis}

In this section, the POD performances of the onboard BDS and GPS data are presented. Firstly, the POD strategy is discussed in the context of dynamic models as well as observation models. The GPS-based POD is evaluated by residual analysis and overlap comparisons. By treating the GPS-based orbits as references, the precision of the GPS and BDS combined POD as well as BDS-based POD with different strategies is evaluated and analyzed.

\subsection{POD strategy}

The precision of dynamic orbit determination is related to the dynamic models as well as the observation models. The dynamic configurations mainly include the arc length for orbit determination, dynamic perturbation models and estimation interval for piecewise dynamic parameters. Gravitational dynamic errors can be minimized by adopting the background model as accurately as possible. However, the non-gravitational perturbations are usually calculated with empirical models with very limited accuracy, which is due to the complexity in modeling the atmosphere density as well as the interactions between satellite surfaces and photons and atmosphere particles. The observation model includes the error models of onboard GNSS observations as well as weighting strategy.

Table 1 lists the detailed strategy for both GPS-based POD, GPS and BDS combined POD and BDS-based POD. A typical arc length of $30 \mathrm{~h}$ from 21:00 on the previous day to 03:00 on the next day is used for POD calculations in our experience, which allows for orbit overlap comparisons between consecutive orbit solutions. Both static field and time-varying gravity are calculated by the state-of-the-art earth gravity model EIGEN-06C (Förste et al. 2011) truncated to degree and order 120 and 50, respectively. The FES2004 model (Lyard et al. 2006) is used for calculating the ocean tide, while models from the IERS Conventions 2003 (McCarthy and Petit 2003) are used for solid earth tide and pole tide perturbations as well as relativistic effects. Non-gravitational perturbations include surface forces such as atmosphere drag and solar radiation pressure (SRP). The box-wing model is used for calculating the SRP perturbation. Since the detailed geometry model of FY-3C is not available yet, a simple geometry model is developed from the satellite platform information with default reflectivity coefficients. This geometry model is also adopted for the drag acceleration calculation, during which the atmosphere density is computed using the DTM94 model (density temperature model; Berger et al. 1998). Empirical accelerations in the along-track and cross-track components are estimated as sine and cosine functions related to the argument of latitude to compensate for dynamic errors.

It should be noted that both drag coefficients and empirical accelerations are estimated as piecewise constant parameters with pre-determined intervals. Empirically, they are often estimated per each orbit revolution since the mismodeled or un-modeled accelerations acting on the satellite are usually in this frequency. In our POD calculations, the drag coefficients and empirical accelerations are estimated every four cycles, i.e., $360 \mathrm{~min}$.

The ionosphere-free carrier phase linear combination (LC) and code range linear combination (PC) are formed as basic observation models, allowing for elimination of the 
Fig. 5 BDS and GPS multipath map estimated as piecewise linear model with grid resolution of $2^{\circ} \times 2^{\circ}$ (unit: $\mathrm{m}$ ). Left panels are estimated MP1 maps while right panels are MP2 maps. From up to bottom the panels are in sequence BDS GEO, BDS IGSO, BDS MEO and GPS multipath maps, respectively
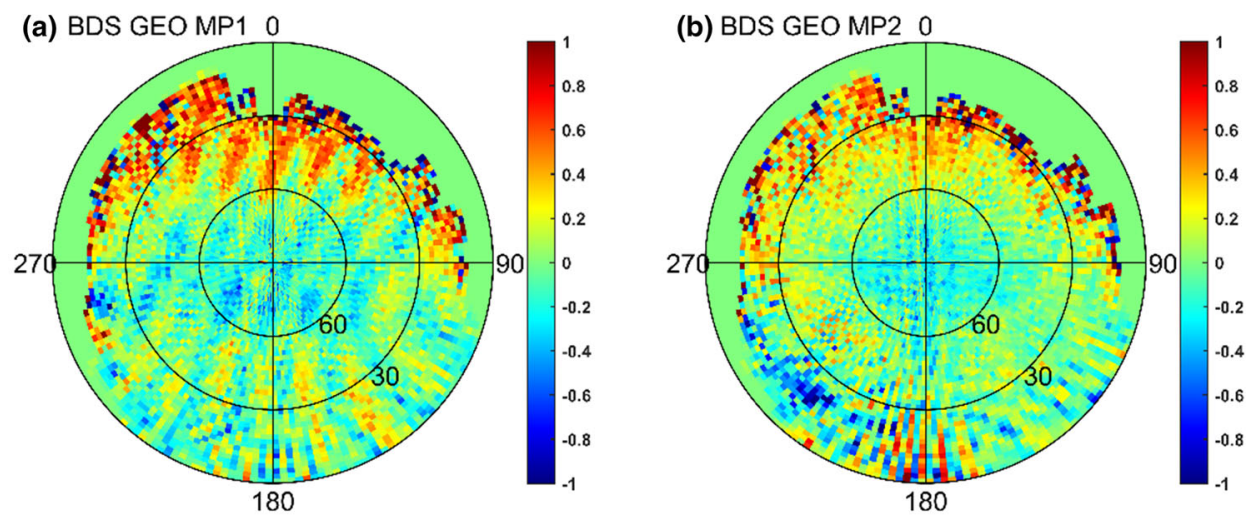

(c) BDS IGSO MP1 0

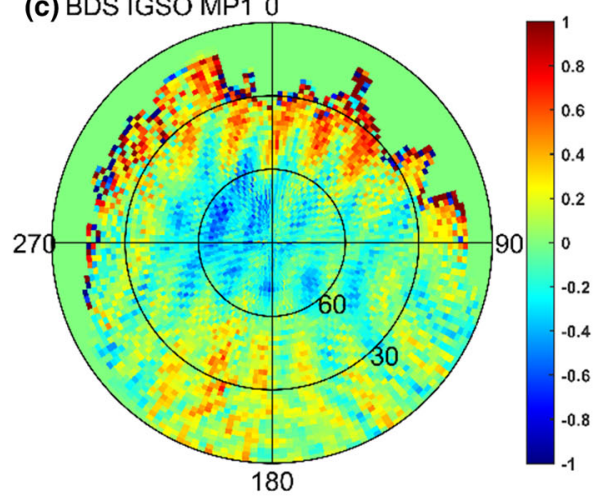

(e) BDS MEO MP1 0
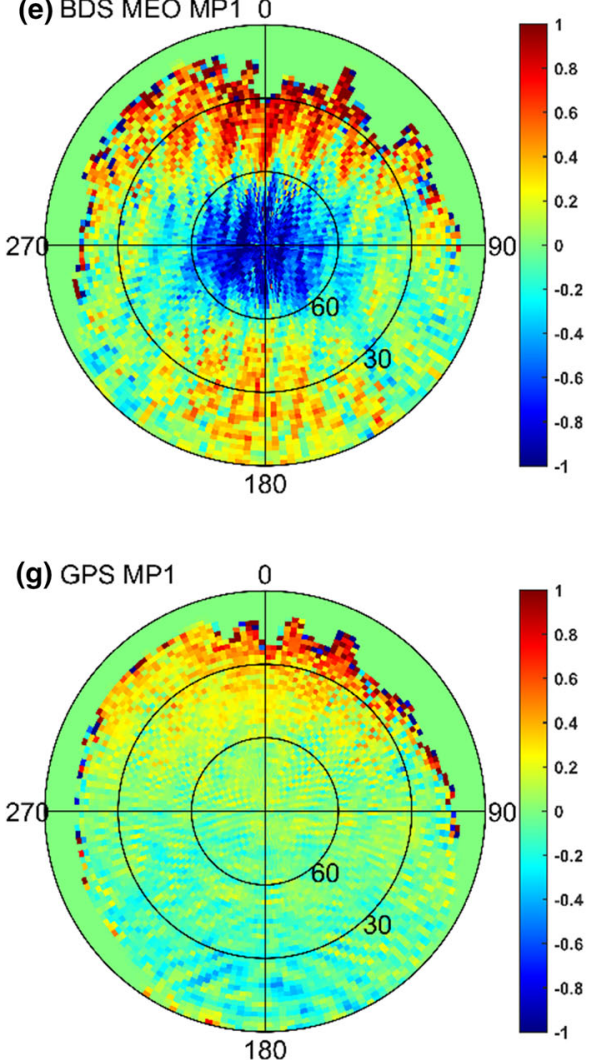
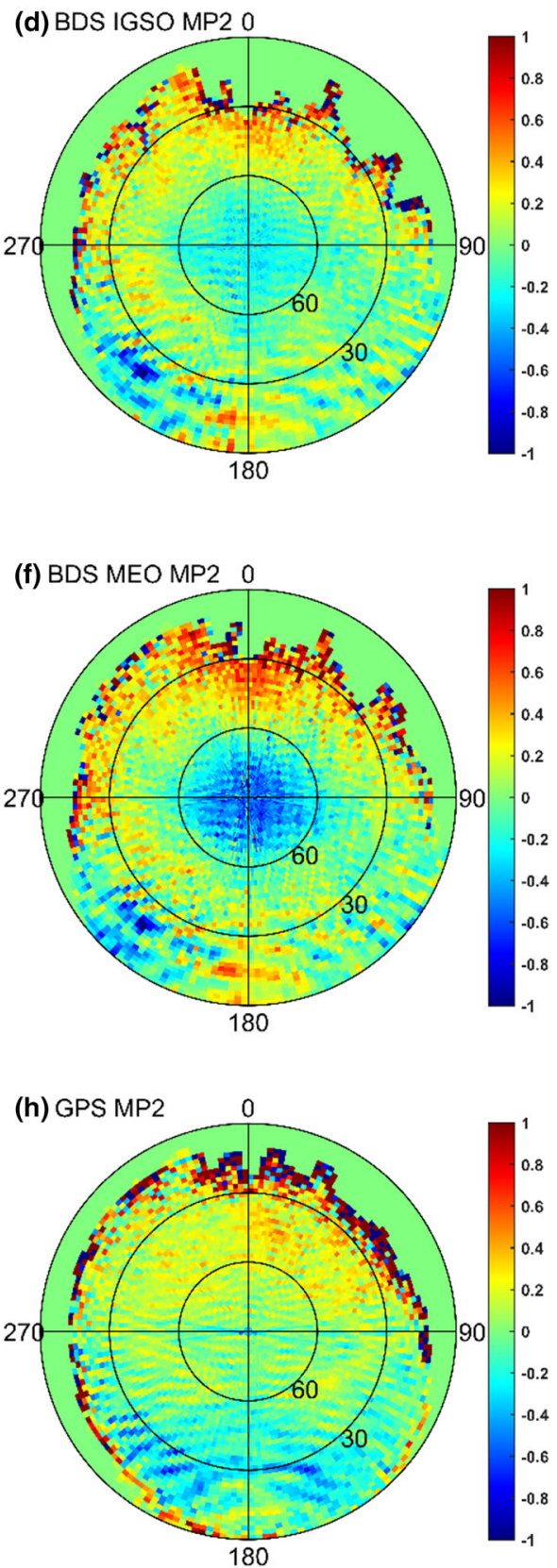
Table 1 GPS/BDS-based FY-3C POD strategy

Reference frame
Conventional inertial reference frame
Precession and nutation
Earth orientation
Dynamic models
Gravity model

Solid earth tide and pole tide

Ocean tide

$\mathrm{N}$-body perturbation

Relativity

Solar radiation pressure

Atmosphere drag

Empirical forces

Observation model

Observation

Arc length and interval

GPS ephemeris and clock

BDS ephemeris and clock

Ionosphere delay

GPS satellite antenna PCO /PCV

BDS satellite antenna PCO /PCV

GNOS PA PCO

GNOS PA PCV
Relativistic effect
Estimated parameters
FY-3C initial state
Receiver clock error
Ambiguities
Drag coefficients
Empirical accelerations

GNOS PA PCV

Relativistic effect

Estimated parameters

FY-3C initial state
$\mathrm{J} 2000.0$

IERS 2003

IERS C-04 (Gambis 2004)

EIGEN-06C, $120 \times 120$ degree and order for static field and $50 \times 50$ degree and order for time-varying gravity

IERS 2003

FES2004 $30 \times 30$

JPL DE405 (Standish 1998)

IERS 2003

Box-wing model

DTM94, piecewise drag coefficients estimated

Piecewise periodical terms in along-track and cross-track components (a priori sigma $10 \mathrm{~nm} / \mathrm{s}^{2}$ )

PC (a priori sigma $3 \mathrm{~m}$ ), LC (a priori sigma $1 \mathrm{~cm}$ )

30 h, $30 \mathrm{~s}$

IGS final precise products (30-s interval for clock products) (Dow et al. 2009)

Recomputed BDS products (30-s interval for clock products)

First-order delay eliminated by ionosphere-free linear combination, higher orders are neglected

IGS ATX model (Schmid et al. 2016)

model from Guo et al. (2016b)

Corrected using default values; $\mathrm{Z}$ component estimated for GPS-based POD

Not considered

IERS Conventions 2003

Position and velocity at initial epoch

One per epoch as process noise

One per satellite pass

One per $360 \mathrm{~min}$

One set per $360 \mathrm{~min}$ first-order ionospheric delay error. Other observation errors including relativistic effects, phase windup and antenna phase center offsets and variations are also considered in observation linearization by using empirical models or theoretical equations (see Hofmann-Wellenhof et al. 2012). In GPS POD, the PCO at the GNOS antenna is firstly corrected by the preliminary values provided in Sect. 2.2 and its $\mathrm{Z}$ component (radial direction) is estimated and calibrated. The estimated PCO Z component is further applied in GPS and BDS combined POD as well as BDS POD. The PCVs (phase center variation) at GNOS antenna are neglected. For GPS satellites, their PCOs and PCVs are corrected using the latest IGS antenna models, while for BDS satellites the PCO and PCV models from Guo et al. (2016b) are utilized, thus consistent with the models adopted for GPS and BDS precision product calculation.

\subsection{GPS-based POD results}

\subsubsection{Residual analysis}

For POD calculations, most observation errors are corrected during the observation linearization step before normal equation stacking, while the un- or mismodeled errors, such as observation noise, are left in the observation equation. From a statistical perspective, part of these errors will be absorbed into the parameters through the estimator, while the rest will still be present in the residuals. Usually large residuals often indicate poor POD estimation. However, small residuals can still be obtained even if some errors are not very well modeled, since they can be absorbed into the parameters during the adjustment process. Therefore, residual analysis can only indicate the internal consistency of POD results but not an external validation.

Figure 6 shows the RMS statistics of the GPS LC and $\mathrm{PC}$ residuals for each of the POD arcs. It can be seen that the LC RMS errors are generally within $10 \mathrm{~mm}$ and the PC RMS within $2.0 \mathrm{~m}$, indicating that the GPS observations are modeled very precisely. The variations in the residual RMS are rather stable from arc to arc for both LC and PC, except a noticeably larger LC RMS for arc on 075/2015 (14.2 mm). RMS of the one-month PC and LC residuals is $9.4 \mathrm{~mm}$ and $1.85 \mathrm{~m}$, respectively.

The statistics for the PC residuals are larger than the RMS value of the PC multipath error as given in Sect. 3.3. The main reason for this is that some mis- or un-modeled errors could easily be left in the residuals and then result in larger $\mathrm{PC}$ residuals, e.g., the ignored $\mathrm{P} 1-\mathrm{C} 1$ code bias in GPS satellites. However, these errors could be easily eliminated in the multipath calculations since a constant bias at each satellite pass is subtracted to account for phase ambiguity; the average part of the multipath errors could be removed from the subtraction simultaneously. 

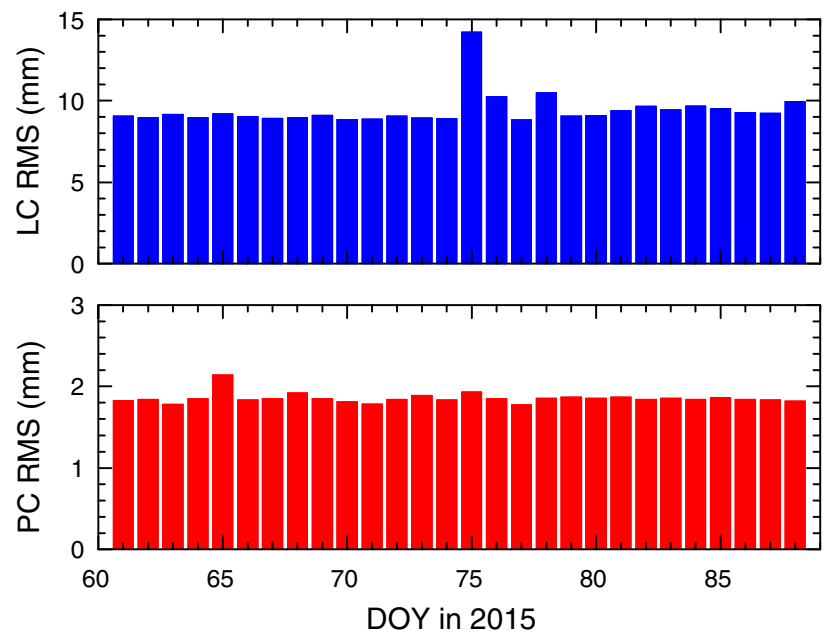

Fig. 6 RMS of GPS LC and PC residuals of each POD arc. Upper panel: LC residuals RMS; Bottom panel: PC residuals RMS

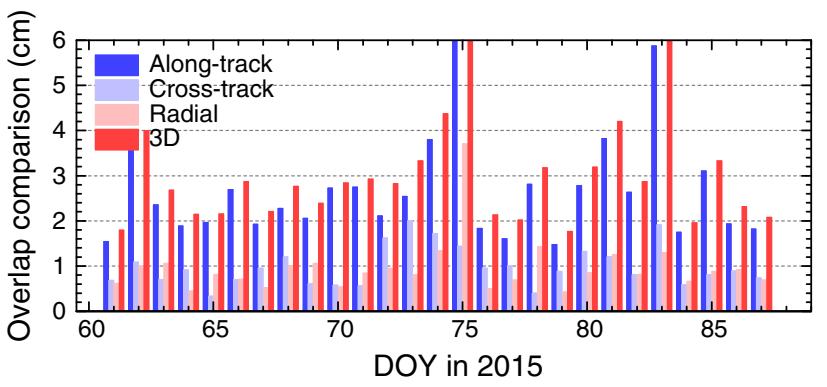

Fig. 7 RMS statistics of the GPS-based orbit overlap differences in the along-track, cross-track and radial directions. The RMS of the alongtrack component on DOY 075 is too large to show in the plot and is $15.88 \mathrm{~cm}$

\subsubsection{Overlap comparison}

The orbit overlap comparison is also widely adopted for orbit precision validation. For the GPS-based POD, there is a $6 \mathrm{~h}$ overlap between two consecutive orbit solutions. The orbit differences in the overlap can be calculated as indicators of orbit quality. Figure 7 indicates the RMS statistics of the GPS-based orbit overlap differences in the along-track (A), cross-track $(\mathrm{C})$ and radial $(\mathrm{R})$ directions. The RMS values of the orbit differences in the along-track component are generally within $3.0 \mathrm{~cm}$ for each overlap, while the radial and cross-track components within $1.0 \mathrm{~cm}$, excepting the large orbit discrepancies for the POD arcs on DOY 075/076 and $083 / 084$. The 3D RMS errors for these two overlaps are 16.37 and $6.31 \mathrm{~cm}$, respectively. The poor orbit overlap precision for arcs on DOY 075/076 may be related to the data quality degradation, as indicated by both the multipath error and the LC residual analysis from Fig. 6. For the overlap on DOY $083 / 084$, the probable cause might be the data gap from 01:33 to 02:55 on DOY 084, as suggested by the exceptionally larger formal errors of the estimated piecewise dynamic
Table 2 Average RMS of orbit overlap differences in the along-track, cross-track, radial and 3D directions

\begin{tabular}{lllll}
\hline & $\begin{array}{l}\text { Along- } \\
\text { track }(\mathrm{cm})\end{array}$ & $\begin{array}{l}\text { Cross- } \\
\text { track }(\mathrm{cm})\end{array}$ & $\begin{array}{l}\text { Radial } \\
(\mathrm{cm})\end{array}$ & 3D $(\mathrm{cm})$ \\
\hline Case A & 3.02 & 0.98 & 0.96 & 3.37 \\
Case B & 2.40 & 0.93 & 0.83 & 2.73 \\
\hline
\end{tabular}

Case A: all arcs are involved in the statistics; Case B: the overlaps on 075/076 and 083/084 are excluded

parameters during that period. For all overlaps, the orbit differences in the along-track component are much larger than the other two components.

The average RMS of the orbit overlap comparisons in each of the three directions as well as position errors (3D RMS) is shown in Table 2. In Case A, all overlaps are involved in the statistics, and the average RMS errors in along-track, cross-track, radial and 3D direction are 3.02, 0.98, 0.96 and $3.37 \mathrm{~cm}$, while in Case B when the overlaps of 075/076 and 083/084 are excluded, the errors are 2.40, 0.93, 0.83 and 2.73 $\mathrm{cm}$, respectively. These overlap comparison results indicate that the GPS-based orbit solutions obtained in this study comprise good internal consistency and that their precision can reach $2-3 \mathrm{~cm}$ level in the 3D RMS, provided that some poorestimated arcs are excluded.

\subsection{BDS-based POD results}

In this section, the POD performance with only onboard BDS data for FY-3C is presented. As indicated in Sect. 3.1, the precision of BDS products especially for GEOs is still limited. Zhao et al. (2015) investigated the BDS PPP performance with BDS GEOs included and excluded, and found that in some cases BDS GEOs could slightly degrade the position accuracy. Hence, two schemes have been explored for BDSbased POD: with BDS GEOs (with GEOs) and without BDS GEOs (w/o GEOs). Based on this, the contribution of the BDS GEOs to the BDS-based POD can be analyzed.

The BDS-based orbits are obtained by POD using above two different strategies. Firstly their LC and PC residuals are analyzed and shown in Fig. 8. The LC residuals for $\mathrm{C} 02$ and $\mathrm{C} 03$ are exceptionally larger than other BDS satellites when the GEOs are taken into the POD calculations, and their RMS errors are 36.2 and $80.0 \mathrm{~mm}$, respectively, while for IGSOs and MEOs the LC residuals RMS errors are within 10-20 mm. However, when BDS GEOs are excluded, the RMS errors of IGSOs and MEOs LC residuals all reduce to around $6 \mathrm{~mm}$. This indicates that the BDS IGSOs' and MEOs' observations can actually be modeled as precisely as the GPS observations. Moreover, though inclusion of GEOs could increase the observation number by roughly $30 \%$ for each arc, their large observation model errors degrade the POD precision seriously, resulting in overall larger LC resid- 


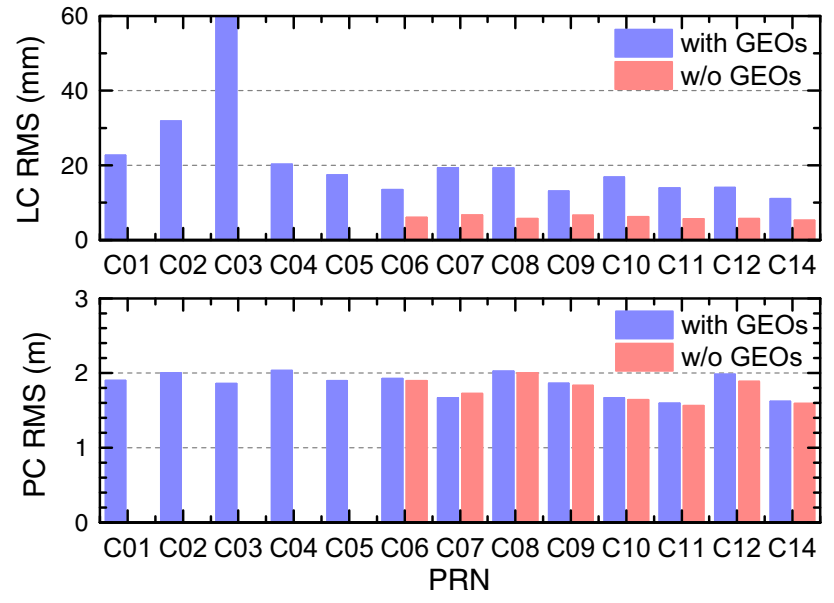

Fig. 8 Average RMS of LC and PC residuals for each BDS satellite. Residuals from all arcs are included in the statistics

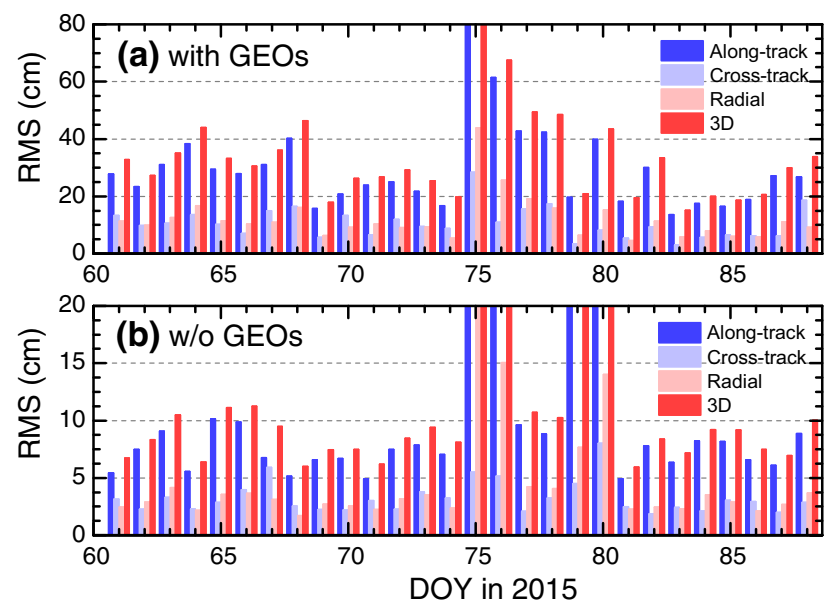

Fig. 9 RMS statistics of the BDS-based orbit differences in the alongtrack, cross-track, radial and 3D directions with respect to the GPSbased orbit solutions

uals. The RMS values of PC residuals of both strategies are generally around $1.8 \mathrm{~m}$, but small improvements can still be observed when GEOs are discarded. The average LC and PC RMS values are listed in Table 3. It is indicated that for the first scheme, the RMS of LC and PC residuals are $22.8 \mathrm{~mm}$ and $1.85 \mathrm{~m}$, respectively, while for the second scheme $6.6 \mathrm{~mm}$ and $1.77 \mathrm{~m}$. It should be noted that in the both cases the average BDS PC residual RMS errors are even smaller than BDS MPC, which suggests the BDS code correction model in Sect. 3.3 effectively improves the accuracy of BDS code observations.

Figure 9 indicates the RMS statistics of the orbit differences between the BDS POD and GPS POD in along-track, cross-track, radial and 3D directions. Before analyzing the different performances of the two strategies, it is observed that the POD results of several arcs are exceptionally worse than the normal ones, i.e., the arcs on DOY 075, 076, 079 and
080. For arcs on DOY 075 and 076 , it should be attributed to poor data quality, as there are no usable data during 11:35 on DOY 075/2015-02:40 on DOY 076/2015. For arcs on DOY 079 and 080 , there is also severe data loss during 15:24 DOY 080/2015-03:10 DOY 081/2015. These 4 arcs are thus excluded from the orbit evaluation analysis.

For the first scheme when BDS GEOs are involved in BDS-based POD, the RMS errors in the along-track component are generally around $30 \mathrm{~cm}$, while the RMS in the cross-track and radial components is much smaller (around $10 \mathrm{~cm}$ ). For the second scheme, the RMS in along-track is mainly within $10 \mathrm{~cm}$, while in cross-track and radial components around $3 \mathrm{~cm}$, showing significant improvements in all three directions compared to the first case. The average RMS errors of the BDS-based POD are calculated and indicated in Table 3, along with the overlap comparison results. The RMS errors in along-track, cross-track and radial component for the first scheme with GEOs are 26.36, 9.88 and 10.25 $\mathrm{cm}$, respectively, while for the second scheme they are 7.31, 2.83 and $2.92 \mathrm{~cm}$, respectively. Their orbit position errors are 30.12 and $8.41 \mathrm{~cm}$, respectively. The results of overlap comparisons are consistent with orbit comparisons, showing 3D RMS errors of 32.67 and $9.98 \mathrm{~cm}$ for the two schemes, respectively.

Above results on BDS-based POD are very inspiring as it achieves precision better than $10 \mathrm{~cm}$ in 3D RMS with just five BDS IGSOs and three MEOs. The good performance of BDS POD for FY-3C should be primarily due to the stable attitude control and dynamic condition of the satellite platform. It is also indicated that inclusion of the BDS GEOs degrades the precision severely, which should be mainly attributed to the large errors of BDS GEO orbit and clock products. It should also be noticed that the amount of BDS observations provided by GNOS receiver is far less than expected especially in the AsiaPacific region due to its channel limits for BDS signals. Hence, better results of BDS-based POD can be obtained if more observations are generated without such restrictions.

\subsection{GPS and BDS combined POD results}

For GPS and BDS combined POD, the orbit quality could be degraded if the BDS data are not handled properly, especially for the GEO observations as their orbit errors are much larger. As indicated by BDS-based POD, inclusion of GEOs results in significant precision degradation of the orbit estimates. Hence, the combined POD calculations are carried out using two different cases: first with BDS GEOs and second without BDS GEOs.

The involvement of the BDS data in the combined POD depends on the BDS observation number and observation weighting. The less the BDS data are involved in the com- 
Table 3 RMS statistics of LC and PC residuals of BDS POD and average RMS of the orbit differences between BDS-based POD and GPS POD

\begin{tabular}{|c|c|c|c|c|c|c|c|c|c|c|}
\hline & \multirow[t]{2}{*}{ LC RMS (mm) } & \multirow[t]{2}{*}{ PC RMS (m) } & \multicolumn{4}{|c|}{ Orbit comparison $(\mathrm{cm})$} & \multicolumn{4}{|c|}{ Orbit overlap $(\mathrm{cm})$} \\
\hline & & & $\mathrm{A}$ & $\mathrm{C}$ & $\mathrm{R}$ & $3 \mathrm{D}$ & $\mathrm{A}$ & $\mathrm{C}$ & $\mathrm{R}$ & $3 \mathrm{D}$ \\
\hline With GEOs & 22.4 & 1.84 & 26.36 & 9.88 & 10.25 & 30.12 & 28.10 & 10.97 & 10.96 & 32.67 \\
\hline W/o GEOs & 6.0 & 1.77 & 7.31 & 2.83 & 2.92 & 8.41 & 8.54 & 3.38 & 3.37 & 9.98 \\
\hline
\end{tabular}
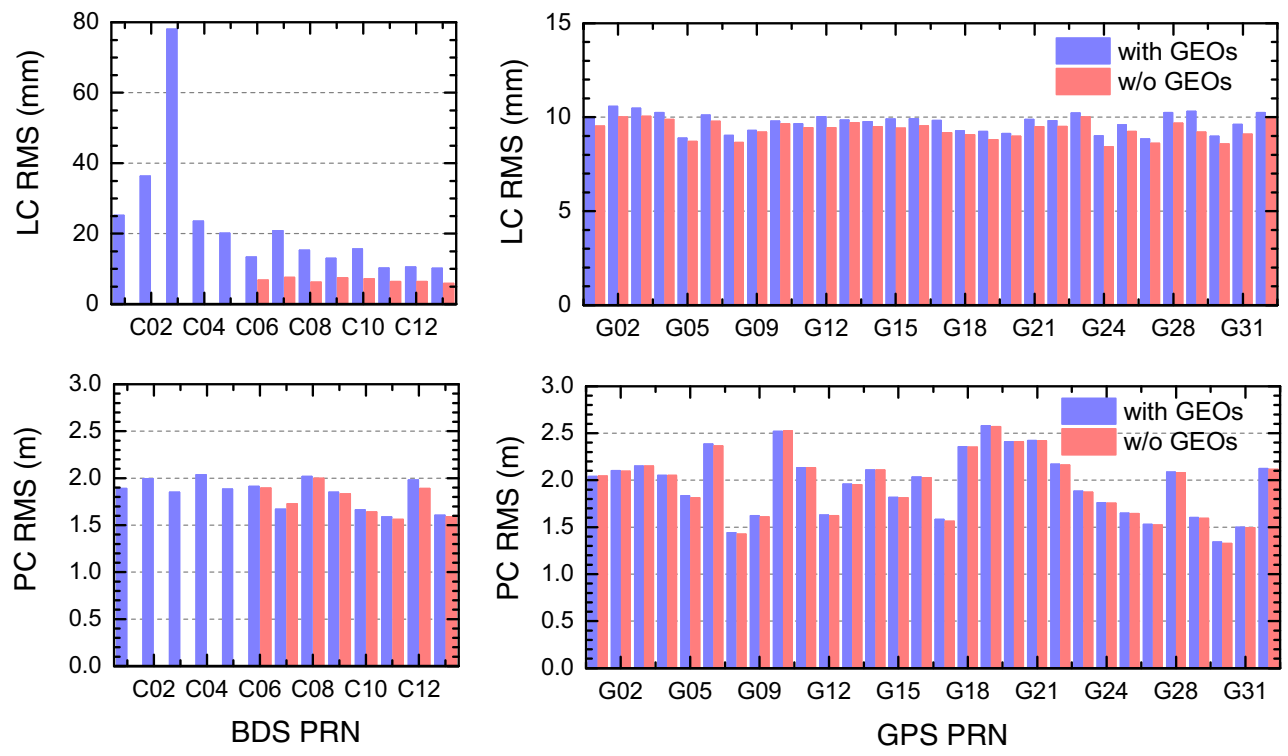

Fig. 10 RMS of GPS and BDS LC (up panels) and PC (bottom panels) residuals from GPS and BDS combined POD

bined POD calculation, the closer the orbit estimation should be with respect to the GPS-only POD. On average, there are about 20000 GPS observations involved for each POD arc, while for BDS the observation number is about 7300 when all BDS satellites are included and about 5400 when BDS GEOs are not used. As the amount of GPS data is already nearly 3-4 times more than that of the BDS data, same a prior precisions for GPS and BDS observations are used to ensure that the BDS data can effectively contribute to the combined POD processing.

Residual analysis and overlap comparison are used for orbit quality assessment as well. As seen in Fig. 10, the average RMS of the LC and PC residuals of combined POD is calculated for each individual satellite for the entire month. In the first case with GEOs included in the combined POD processing, the LC residual RMS values of BDS GEOs are exceptionally larger than the IGSOs and MEOs, and the RMS errors of $\mathrm{C} 02$ and $\mathrm{C} 03$ satellites even reach to 36 and $77 \mathrm{~mm}$, respectively, which is very similar with the first case of BDSbased POD. This should be attributed to the degraded orbit precision of the BDS GEOs as mentioned earlier. The GPS LC residuals are very close to the GPS-based POD, all in the vicinity of $10 \mathrm{~mm}$. The RMS of PC residuals for BDS and GPS are around $2.0 \mathrm{~m}$. However, in the second case without GEOs, the BDS LC residuals all reduce to below $8 \mathrm{~mm}$, and there are overall slight improvements for GPS LC residuals as well. This again indicates that the observation model errors of BDS GEOs degrade the precision of POD severely. The average BDS and GPS LC and PC residual RMS errors are calculated and listed in Table 4. The RMS improvements of the BDS LC and PC residuals of the second case against the first are $15.7 \mathrm{~mm}$ and $0.07 \mathrm{~m}$, respectively. The RMS errors of BDS PC and LC residuals in both cases are very consistent with those obtained in BDS-based POD as shown in Table 3.

Orbit overlap comparisons of the first and second cases are shown in Fig. 11a and b, respectively. The orbit overlap differences are much larger in the first case, and their RMS values are generally around $4 \mathrm{~cm}$. Comparatively, the overlap differences in the second case are nearly the same with respect to the GPS-based POD, showing 3D RMS errors around $3 \mathrm{~cm}$ and exceptionally larger for arc on DOY 075. Particularly, the large overlap differences for arc DOY 083 in GPS POD have been reduced to $4.69 \mathrm{~cm}$ (3D RMS), which is attributed to that there are 1-3 BDS observations per epoch during the GPS data gap mentioned in Sect. 4.2. The combined POD results are also compared to the GPS-based POD results by treating the GPS-derived orbits as reference orbits. Their orbit differences are calculated and shown in Fig. 11c and $\mathrm{d}$ for the two cases, respectively. For both cases, the 
Table 4 Statistics of average LC and PC RMS, orbit overlap RMS of combined POD as well as orbit difference RMS between combined POD and GPS POD in along-track, cross-track, radial and 3D components

\begin{tabular}{|c|c|c|c|c|c|c|c|c|c|c|c|c|}
\hline & \multicolumn{2}{|c|}{ LC RMS (mm) } & \multicolumn{2}{|c|}{ PC RMS (m) } & \multicolumn{4}{|c|}{ Orbit overlaps $(\mathrm{cm})$} & \multicolumn{4}{|c|}{ Orbit comparison $(\mathrm{cm})$} \\
\hline & BDS & GPS & BDS & GPS & A & $\mathrm{C}$ & $\mathrm{R}$ & $3 \mathrm{D}$ & A & $\mathrm{C}$ & $\mathrm{R}$ & $3 \mathrm{D}$ \\
\hline With GEOs & 22.4 & 9.7 & 1.84 & 1.96 & 3.44 & 1.15 & 1.23 & 3.86 & 3.02 & 1.11 & 1.13 & 3.43 \\
\hline W/o GEOs & 6.7 & 9.3 & 1.77 & 1.95 & 2.45 & 0.79 & 0.83 & 2.73 & 0.66 & 0.22 & 0.27 & 0.75 \\
\hline
\end{tabular}

(a)

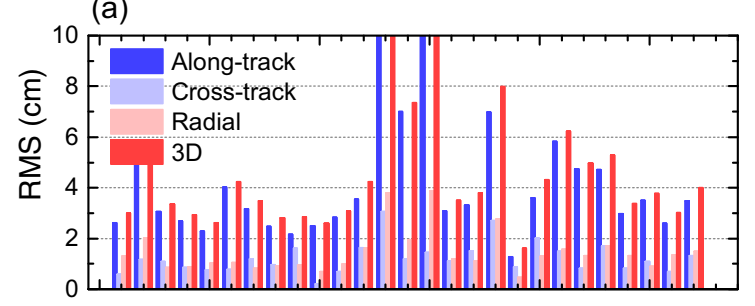

(c)

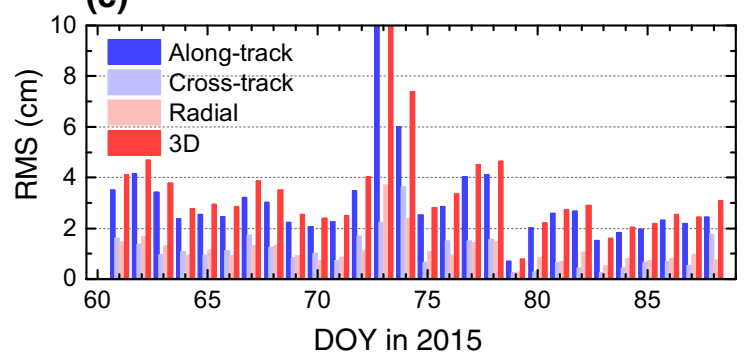

Fig. 11 RMS of orbit overlap differences of combined POD (up panels) and orbit differences between combined POD and GPS POD (bottom panels). a Overlap differences of combined POD with BDS GEOs; b overlap differences of combined POD without BDS GEOs; $\mathbf{c}$

RMS errors of orbit comparisons to GPS-derived orbits are roughly consistent with the results of overlap comparisons but are overall smaller, which is because that overlap comparison usually overestimates the orbit errors due to the edge effect. For the second case, the RMS errors are generally below $1 \mathrm{~cm}$, indicating that the combined POD results are very close to the GPS-based POD.

The average RMS values of orbit overlap differences and orbit comparison to GPS-based POD are listed in Table 4. For the first case, the orbit precision is at $3.5 \mathrm{~cm}$ level by both overlap comparison and orbit comparison; however, when BDS GEOs are excluded, the orbit precision is nearly the same with GPS-only POD. The overlap differences in alongtrack, cross-track, radial and 3D components are 2.45, 0.79, 0.83 and $2.73 \mathrm{~cm}$, respectively, while orbit differences comparing to GPS-derived orbits are only $0.75 \mathrm{~cm}$ in 3D RMS. Both BDS-based POD and combined POD demonstrate that BDS GEOs degrade the POD processing precision. Since GPS data dominate the combined POD performance, the precision degradation of combined POD is much smaller than BDS-based POD. (b)

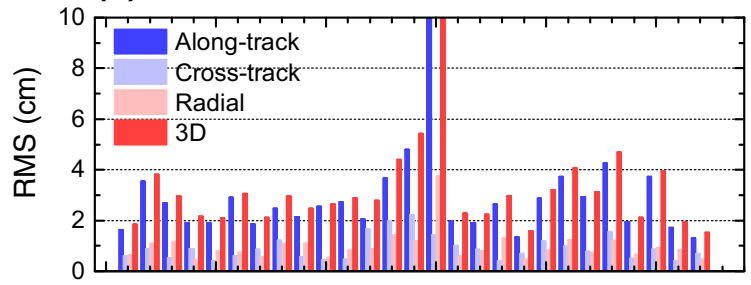

(d)

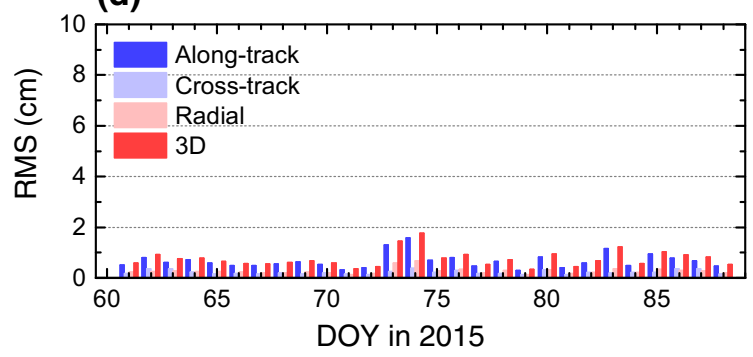

orbit comparison between combined POD with BDS GEOs and GPS POD; $\mathbf{d}$ orbit comparison between combined POD without BDS GEOs and GPS POD

\section{Conclusion}

In this study, BDS and GPS data onboard the Chinese FY-3C satellite during March 2015 are analyzed. Firstly, the BDS and GPS observation quality is evaluated in the context of the data quantity as well as the code range multipath errors. Following this, the FY-3C POD using only GPS, only BDS data, as well as combined GPS and BDS is performed and their POD precisions are analyzed.

For the onboard BDS data quality, the data amount is mainly limited by the configuration of the BDS constellations, as well as maximum channels allocated for BDS signals of the onboard GNOS instrument. There are about $17 \%$ epochs with no available data at all and only $30-40 \%$ with four to six satellites. Importantly, about $75 \%$ of observations are distributed in the Eastern Hemisphere. The BDS MP1, MP2 and MPC RMS errors are 0.73, 0.67 and $2.03 \mathrm{~m}$, respectively. The onboard BDS data shows significant multipath errors associated with elevation and azimuth, and large multipath biases are observed for BDS MEOs when elevation is over $60^{\circ}$. In comparison, there are $88 \%$ epochs with more 
than four useful GPS satellites, and the GPS MP1, MP2 and MPC RMS are 0.36, 0.61 and $1.29 \mathrm{~m}$, respectively.

The orbit precision of GPS-based POD is evaluated by residual analysis and overlap comparison, showing good orbit consistency of $2.73 \mathrm{~cm}$ in 3D RMS. The GPS-derived orbits are used as reference to assess the precision of the BDS-based POD as well as GPS and BDS combined POD. Results from both BDS-based POD and combined POD indicate that inclusion of the BDS GEOs could lead to severe orbit estimation degradation. When BDS GEOs are excluded, the orbit precision using BDS data alone can reach $10 \mathrm{~cm}$ level in 3D RMS, while for GPS and BDS combined POD the orbit differences compared to GPS-derived orbits are only 0.75 $\mathrm{cm}$.

The results indicate that the precision of LEO POD using onboard BDS data alone could reach $3.0 \mathrm{~cm}$ level in the radial component and better than $10 \mathrm{~cm}$ in 3D RMS, even though only five BDS IGSOs and three MEOs are involved. This precision already satisfies the requirements of most remote sensing missions. Since more BDS satellites will come into service and the number of ground track stations is increasing steadily, there is opportunity for the quality of BDS precision products as well as onboard BDS performances to be further improved.

Acknowledgements We are very grateful to the IGS for providing the GPS precise orbit and clock products and Dr. Jing Guo for providing the BDS orbit and 30-s clock error products. We would like to thank Dr. Adrian Jäggi and three anonymous reviewers for their valuable comments and suggestions. This work is sponsored by the National Key Research and Development Program of China (Grant No. 2016YFB0501800), Natural Science Foundation of China (Grant No. 41574027, 41325015, 41574030, 41505030) and Natural Science Key Program of Hubei Province, China (Grant No. 2015CFA057).

\section{Compliance with ethical standards}

Conflicts of interest The authors declare they have no conflicts of interest.

Open Access This article is distributed under the terms of the Creative Commons Attribution 4.0 International License (http://creativecomm ons.org/licenses/by/4.0/), which permits unrestricted use, distribution, and reproduction in any medium, provided you give appropriate credit to the original author(s) and the source, provide a link to the Creative Commons license, and indicate if changes were made.

\section{References}

Bai W, Sun Y, Du Q, Yang G, Yang Z, Zhang P, Bi Y, Wang X, Cheng C, Han Y (2014) An introduction to the FY3 GNOS instrument and mountain-top tests. Atmos Meas Tech 7:1817-1823. doi:10. 5194/amt-7-1817-2014

Berger C, Biancale R, Ill M, Barlier F (1998) Improvement of the empirical thermospheric model DTM: DTM94-a comparative review of various temporal variations and prospects in space geodesy applications. J Geodes 72:161-178
Bertiger W, Desai SD, Dorsey A, Haines BJ, Harvey N, Kuang D, Sibthorpe A, Weiss JP (2010) Sub-centimeter precision orbit determination with GPS for ocean altimetry. Mar Geodes 33:363-378. doi:10.1080/01490419.2010.487800

Bi Y, Yang Z, Zhang P, Sun Y, Bai W, Du Q, Yang G, Chen J, Liao M (2012) An introduction to China FY3 radio occultation mission and its measurement simulation. Adv Space Res 49:1191-1197. doi:10.1016/j.asr.2012.01.014

Bock H, Jäggi A, Beutler G, Meyer U (2014) GOCE: precise orbit determination for the entire mission. J Geodes 88:1047-1060. doi:10. 1007/s00190-014-0742-8

Cai C, Gao Y, Pan L, Zhu J (2015) Precise point positioning with quadconstellations: GPS, BeiDou, GLONASS and Galileo. Adv Space Res 56:133-143. doi:10.1016/j.asr.2015.04.001

Chen X, Zhao S, Wang M, Lu M (2016) Space-borne BDS receiver for LING QIAO satellite: design, implementation and preliminary in-orbit experiment results. GPS Solut 20:837-847. doi:10.1007/ s10291-015-0493-x

Dai X, Ge M, Lou Y, Shi C, Wickert J, Schuh H (2015) Estimating the yaw-attitude of BDS IGSO and MEO satellites. J Geodes 89:10051018. doi:10.1007/s00190-015-0829-x

Deng Z, Ge M, Uhlemann M, Zhao Q (2014) Precise orbit determination of BeiDou satellites at GFZ. In: Proceedings of IGS workshop, pp $23-27$

Dow J, Neilan R, Rizos C (2009) The international GNSS service in a changing landscape of global navigation satellite systems. J Geodes 83:191-198. doi:10.1007/s00190-008-0300-3

Förste C, Bruinsma S, Shako R, Marty J-C, Flechtner F, Abrykosov O, Dahle C, Lemoine J-M, Neumayer K-H, Biancale R, EIGEN Team (2011) EIGEN-6 - A new combined global gravity field model including GOCE data from the collaboration of GFZ Potsdam and GRGS Toulouse (Geophysical Research Abstracts Vol. 13, EGU2011-3242-2, 2011), General Assembly European Geosciences Union, Vienna, Austria

Gambis D (2004) Monitoring earth orientation using space-geodetic techniques: state-of-the-art and prospective. J Geodes 78:295-303. doi:10.1007/s00190-004-0394-1

Guo J, Zhao Q, Guo X, Liu X, Liu J, Zhou Q (2015) Quality assessment of onboard GPS receiver and its combinationwith DORIS and SLR for Haiyang 2A precise orbit determination. Sci China Earth Sci 58:138-150. doi:10.1007/s11430-014-4943-Z

Guo F, Li X, Zhang X, Wang J (2016a) Assessment of precise orbit and clock products for Galileo, BeiDou, and QZSS from IGS Multi-GNSS Experiment (MGEX). GPS Solut. 1-12. doi:10.1007/ s10291-016-0523-3

Guo J, Xu X, Zhao Q, Liu J (2016b) Precise orbit determination for quad-constellation satellites at Wuhan University: strategy, result validation, and comparison. J Geodes 90:143-159. doi:10.1007/ s00190-015-0862-9

Haines B, Bar-Sever Y, Bertiger W, Desai S, Willis P (2004) Onecentimeter orbit determination for Jason-1: new GPS-based strategies. Mar Geodes 27:299-318. doi:10.1080/01490410490465300

Hofmann-Wellenhof B, Lichtenegger H, Collins J (2012) Global positioning system: theory and practice. Springer, Berlin

IGS (2015) RINEX: the receiver independent exchange format (Version 3.03). IGS Central 2015

Kang Z, Tapley B, Bettadpur S, Ries J, Nagel P, Pastor R (2006) Precise orbit determination for the GRACE mission using only GPS data. J Geodes 80:322-331. doi:10.1007/s00190-006-0073-5

Lemoine FG, Zelensky NP, Chinn DS, Pavlis DE, Rowlands DD, Beckley BD, Luthcke SB, Willis P, Ziebart M, Sibthorpe A, Boy JP, Luceri V (2010) Towards development of a consistent orbit series for TOPEX, Jason-1, and Jason-2. Adv Space Res 46:1513-1540. doi:10.1016/j.asr.2010.05.007 
Li M, Qu L, Zhao Q, Guo J, Su X, Li X (2014) Precise point positioning with the BeiDou navigation satellite system. Sensors (Basel) 14:927-943. doi:10.3390/s140100927

Li M, Li W, Shi C, Zhao Q, Su X, Qu L, Liu Z (2015a) Assessment of precipitable water vapor derived from ground-based BeiDou observations with precise point positioning approach. Adv Space Res 55:150-162. doi:10.1016/j.asr.2014.10.010

Li X, Zhang X, Ren X, Fritsche M, Wickert J, Schuh H (2015b) Precise positioning with current multi-constellation global navigation satellite systems: GPS, GLONASS, Galileo and BeiDou. Sci Rep 5:8328. doi: $10.1038 /$ srep08328

Liao M, Zhang P, Yang G-L, Bi Y-M, Liu Y, Bai W-H, Meng X-G, Du QF, Sun Y-Q (2016) Preliminary validation of the refractivity from the new radio occultation sounder GNOS/FY-3C. Atmos Meas Tech 9:781-792. doi:10.5194/amt-9-781-2016

Liu J, Gu D, Ju B, Yao J, Duan X, Yi D (2014) Basic performance of BeiDou-2 navigation satellite system used in LEO satellites precise orbit determination. Chin J Aeronaut 27:1251-1258. doi:10.1016/ j.cja.2014.03.006

Lou Y, Liu Y, Shi C, Yao X, Zheng F (2014) Precise orbit determination of BeiDou constellation based on BETS and MGEX network. Sci Rep 4:4692. doi:10.1038/srep04692

Lu C, Li X, Nilsson T, Ning T, Heinkelmann R, Ge M, Glaser S, Schuh H (2015) Real-time retrieval of precipitable water vapor from GPS and BeiDou observations. J Geodes 89:843-856. doi:10. 1007/s00190-015-0818-0

Lyard F, Lefevre F, Letellier T, Francis O (2006) Modelling the global ocean tides: modern insights from FES2004. Ocean Dyn 56:394415. doi:10.1007/s10236-006-0086-x

McCarthy DD, Petit G (2003) IERS conventions 2003. IERS technical note No. 32. Bundesamt fuer Kartographie und Geodaesie, Frankfurt am Main

Montenbruck O, Kroes R (2003) In-flight performance analysis of the CHAMP BlackJack GPS receiver. GPS Solut 7:74-86. doi:10. 1007/s10291-003-0055-5

Montenbruck O, Garcia-Fernandez M, Williams J (2006) Performance comparison of semicodelessGPS receivers for LEOsatellites.GPS Solut 10:249-261. doi:10.1007/s10291-006-0025-9

Prange L, Dach R, Lutz S, Schaer S, Jäggi A (2015) The CODE MGEX Orbit and Clock Solution. Springer, Berlin, Heidelberg pp 1-7

Rocken C, Meertens C (1992) UNAVCO receiver tests. UNAVCO Memo, Nov 8, 1992

Schmid R, Dach R, Collilieux X, Jäggi A, Schmitz M, Dilssner F (2016) Absolute IGS antenna phase center model igs08.atx: status and potential improvements. J Geodes 90(4):343-364. doi:10.1007/ s00190-015-0876-3

Shi C, Zhao Q, Li M, Tang W, Hu Z, Lou Y, Zhang H, Niu X, Liu J (2012) Precise orbit determination of Beidou satellites with precise positioning. Sci China-Earth Sci 55:1079-1086. doi:10.1007/ s11430-012-4446-8
Standish (1998) JPL Planetary and Lunar Ephemerides, DE405/LE405. JPL Interoffice Memorandum 312.F-98-048

Steigenberger P, Hugentobler U, Hauschild A, Montenbruck O (2013) Orbit and clock analysis of Compass GEO and IGSO satellites. J Geodes 87:515-525. doi:10.1007/s00190-013-0625-4

Tapley BD, Ries J, Davis G, Eanes R, Schutz B, Shum C, Watkins M, Marshall J, Nerem R, Putney B (1994) Precision orbit determination for TOPEX/POSEIDON. J Geophys Res Ocean 99:2438324404

Uhlemann M, Gendt G, Ramatschi M, Deng Z (2015) GFZ Global Multi-GNSS Network and Data Processing Results. In: Rizos C, Willis P (eds) IAG 150 Years. International Association of Geodesy Symposia, vol 143. Springer, Cham

Wang X, Sun Y, Du Q, Bai W, Wang D, Cai Y, Wu D, Yu Q (2014) GNOS-Radio occultation sounder on board of Chinese FY3 satellites. In: 2014 IEEE Geoscience and Remote Sensing Symposium. IEEE, pp 4982-4985

Wanninger L, Beer S (2015) BeiDou satellite-induced code pseudorange variations: diagnosis and therapy. GPS Solut 19:639-648. doi:10.1007/s10291-014-0423-3

Xu A, Xu Z, Ge M, Xu X, Zhu H, Sui X (2013) Estimating zenith tropospheric delays from BeiDou navigation satellite system observations. Sensors 13:4514-4526. doi:10.3390/s130404514

Yang J, Zhang P, Lu N, Yang Z, Shi J, Dong C (2012) Improvements on global meteorological observations from the current Fengyun 3 satellites and beyond. Int J Digit Earth 5:251-265. doi:10.1080/ 17538947.2012.658666

Yang W, Tong H, Pan L, Xu D, Guo W, Yang J (2016) Analysis and correction of BDS code multipath bias. In: Sun J, Liu J, Fan S, Wang F (eds) China Satellite Navigation Conference (CSNC) 2016 Proceedings: Volume III. Lecture notes in Electrical Engineering, vol 390. Springer, Singapore

Zhang R, Song W, Yao Y, Shi C, Lou Y, Yi W (2015) Modeling regional ionospheric delay with ground-based BeiDou and GPS observations in China. GPS Solut 19:649-658. doi:10.1007/ s10291-014-0419-z

Zhao Q, Guo J, Li M, Qu L, Hu Z, Shi C, Liu J (2013) Initial results of precise orbit and clock determination for COMPASS navigation satellite system. J Geodes 87:475-486. doi:10.1007/ s00190-013-0622-7

Zhao Q, Wang C, Guo J, Liu X (2015) Assessment of the contribution of BeiDou GEO, IGSO, and MEO satellites to PPP in Asia-Pacific Region. Sensors 15(12):29970-29983 